\title{
Measurement of $\mathrm{rf}$ voltage in long traveling wave accelerating structures with application to the CERN Super Proton Synchrotron
}

\author{
Patrick Kramere* \\ CERN, 1211 Geneva 23, Switzerland \\ and Institute of High Frequency Technology (IHF), RWTH Aachen University, Germany \\ Christine Vollinger \\ CERN, 1211 Geneva 23, Switzerland
}

(Received 12 August 2019; published 17 October 2019)

\begin{abstract}
Since its inauguration in 1976, the Super Proton Synchrotron (SPS) at CERN relies on a traveling wave system for particle acceleration instead of making use of standing wave cavities that are more common in circular accelerators. These traveling wave structures (TWSs) are essentially periodically loaded waveguides operated at a fundamental frequency of about $200 \mathrm{MHz}$. In the frame of the LHC injector upgrade project, the SPS accelerating system is presently improved to better cope with the increased beam intensity required for the future High Luminosity LHC era. The available accelerating voltage provided by the TWSs by means of radio-frequency (rf) generators is a fundamental and valuable input for machine operation and particle tracking codes used to simulate beam phenomena in the SPS. Measurement of the voltage with well known nonresonant and on-axis perturbation methods is however infeasible due to the extreme mechanical length of the SPS structures. It is shown that a good estimate of the rf voltage can be obtained from off-axis measurement samples in each accelerating gap in addition to the in- and output reflection coefficients of the structures. The rf voltage for the LHC proton beam delivered by the system configuration that was installed during the first and second exploitation run of the LHC is measured by this method, not including the beam loading. The impact on the fundamental accelerating mode and the rf voltage of additional higher order mode (HOM) couplers, which are also required for the upgrade of the acceleration system, is assessed by the proposed method as well.
\end{abstract}

DOI: 10.1103/PhysRevAccelBeams.22.101002

\section{INTRODUCTION}

The complete LHC injector chain at CERN is presently stopped for upgrades to better cope with the increased future beam intensity of $2.4 \times 10^{11}$ protons per bunch required by the High Luminosity LHC [1]. In the SPS, the accelerating traveling wave system is upgraded by an increase of installed $\mathrm{rf}$ power, improved damping of deteriorating longitudinal HOMs and the rearrangement of the TWSs into a configuration that is less susceptible to the increased beam loading at higher intensities [2,3]. TWSs for particle accelerator applications have a long history of research, refer e.g., to [4,5]. The TWSs in the SPS are of the parallel stem drift-tube type [6,7]. A unit-cell of the structure is shown in Fig. 1 and has a length of

\footnotetext{
*patrick.kramer@cern.ch
}

Published by the American Physical Society under the terms of the Creative Commons Attribution 4.0 International license. Further distribution of this work must maintain attribution to the author(s) and the published article's title, journal citation, and DOI.
$0.374 \mathrm{~m}$. The circular waveguide is periodically loaded by drift-tubes and stems suspended on pedestals. Refer to [8] for other cell dimensions. For manufacturing and handling reasons, each TWS consists of 11-cell sections, see Fig. 2.

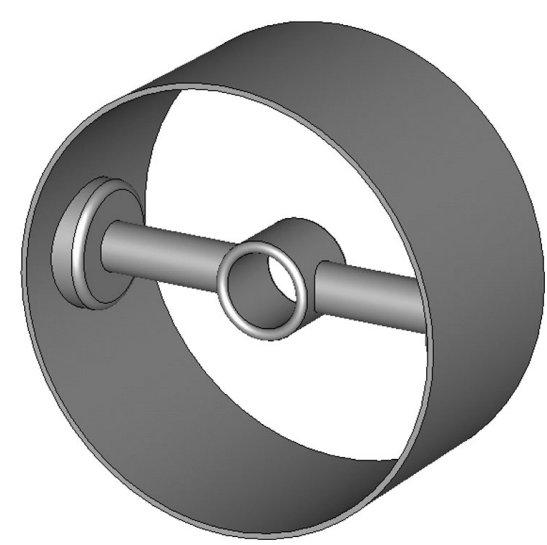

FIG. 1. Unit-cell of SPS parallel stem structure loaded by a drift-tube and stems that are suspended on pedestals. 


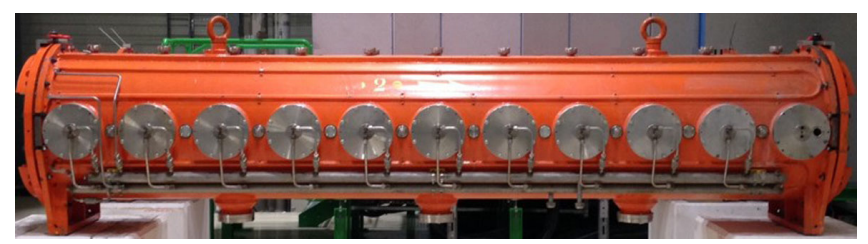

FIG. 2. Eleven-cell section of the TWS with access ports on top of each cell used for HOM-damping, ten lateral ports in each accelerating gap used for low-level rf systems and three vacuum pumping ports at the bottom.

Tuning was carried out once on a per section basis by adjusting the stem length in each cell before brazing [6].

So far, the SPS acceleration system consisted of two 44and two 55-cell structures fed by a peak input power of $1 \mathrm{MW}$ each. The two 55-cell structures are rearranged to four 33-cell structures by adding two 11-cell spare sections that were not deployed until now. The input power for each of the 44- and 33-cell structures will be 1.6 MW and $1.05 \mathrm{MW}$ respectively after the upgrade. The HOMs around $630 \mathrm{MHz}$ in the acceleration system trigger a longitudinal coupled bunch instability at an intensity threshold below the future requirement [3,9]. These HOMs are already heavily mitigated by damping probes installed via access ports available on top of each cell of the periodic structures. However, increased damping by an additional factor of at least three is required to reach High Luminosity LHC goals. Several options solving the numerous obstacles for improved HOM mitigation have been developed in $[10,11]$ and require the placement of additional HOMcouplers in so far unoccupied cells. As also a damping scheme for transverse HOMs is installed, more than $70 \%$ of the cells will be occupied by HOM-couplers after the upgrade. The large number of HOM couplers in the TWSs triggered an in-depth study of the possible deteriorating impact, i.e., detuning or damping, of the fundamental accelerating mode and consequently on the available rf voltage. The TWSs are fixed-tuned to the former transition energy frequency of $200.222 \mathrm{MHz}$, but effectively accelerate the particle beam over a wide frequency band using the $\pi / 2$ traveling-wave mode [6]. The tuning was carried out on the undamped structure, i.e., before the addition of any HOM-couplers. Today, the typical range of rf frequency required for acceleration of the LHC proton beam is from $200.265 \mathrm{MHz}$ at $26 \mathrm{GeV} / \mathrm{c}$ to $200.394 \mathrm{MHz}$ at $450 \mathrm{GeV} / \mathrm{c}$. The fixed target beam is injected at $14 \mathrm{GeV} / \mathrm{c}$ and $199.946 \mathrm{MHz}$ rf frequency. For heavy ions, fixed-frequency acceleration is used in the lowest $\mathrm{rf}$ frequency range.

The rf voltage in the TWSs could in principle be sampled over the frequency range of the fundamental passband (FPB) by a nonresonant, on-axis perturbation method, see, e.g., [12]. This is however impractical for the $200 \mathrm{MHz}$ structure in the SPS firstly due to its extreme length of up to $21 \mathrm{~m}$ in the 55-cell case. Further, the insertion of the wire itself on the axis of the structure is problematic and the procedure risks deterioration of the interior surfaces. Measurement errors due to sagging of the long wire are to be expected as well if this method is deployed. Finally, the TWSs need to be vented and access to the beam vacuum pipes must be ensured.

A good estimate of the rf voltage can however also be obtained without relying on perturbation techniques. The method proposed here, consists of two steps. First, the transit time factor (TTF) of the accelerating field for the particle transit is measured by off-axis probing of the traveling wave in each accelerating gap of the structure. The tuning of the TWS is then directly obtained from the synchronous frequency at which the TTF peaks. In a second step, the absolute voltage is calculated from the ideal case while taking into account the deteriorating mismatch of generator and terminating load at the inand output of the structure. Venting of the TWSs is not necessarily required. The method also takes advantage of the fact that the $\pi / 2$ operating mode in the passband center has a large group velocity and is relatively stable against detuning of individual cells, as could happen e.g., by the insertion of HOM-couplers [8].

The remaining part of this paper is organized as follows. The next section is a brief revision of the forward and beam coupling impedances of TWSs. Section III shows that the TTF of a TWS can be measured via probes in each accelerating gap and the method is applied to the TWSs used in the SPS before the upgrade. The method is then equally used to assess the impact of HOM-couplers on the rf voltage, see Sec. IV. Section V quantifies the impact of a generator and load mismatch on the rf voltage of a TWS and Sec. VI proposes a convenient and fast matching procedure for TWSs fed by transmission lines. Section VII uses the SPS $200 \mathrm{MHz}$ parallel stem drift-tube structure as a simulation example for demonstration of the matching technique and to verify previous findings before these are used in Sec. VIII to estimate the absolute rf voltage delivered by the four TWSs in the SPS before the upgrade. Finally, conclusions are given in Sec. IX.

\section{IMPEDANCES OF TRAVELING WAVE STRUCTURES}

A standing wave cavity presents the identical impedance to the rf generator as well as to the beam. For a TWS, these two differ and are denoted the forward impedance and the beam coupling impedance respectively. In practice, the attenuation length $l_{0}$ of a TWS is much larger than the optimum guide wavelength $l_{\text {opt }}$ for a power efficient operation of the structure at the edges of the usable frequency band $\pm \Delta f / 2$ [13]

$$
\frac{l_{\mathrm{opt}}}{l_{0}}=4.68 \frac{f}{Q \Delta f} .
$$


A typical normal-conducting accelerating structure operated at $f=200 \mathrm{MHz}$ has a quality factor of around 20000 . If as an example the relative frequency swing of $0.44 \%$ required for proton acceleration in the SPS (injection at $10 \mathrm{GeV}$ ) is used, then $l_{\text {opt }} \approx 0.053 l_{0}$. For the following analyses, the TWS can therefore assumed to be lossless.

For effective particle acceleration, the phase velocity $v_{p}$ of the electromagnetic (EM) traveling wave must be equal or close to the particle velocity. Let us assume equal particle and phase velocity for some relative particle velocity $\beta=\beta_{0}$ and traveling wave frequency $\omega=\omega_{0}$. For this case of perfect synchronism the rf voltage is directly proportional to the length of the structure $L$ and given as $V_{r f}=E_{0} L e^{j \Phi}$ with the accelerating electric field $E_{0}$. The angle $\Phi$ accounts for a possible phase shift between particle and wave half-way along the structure due to the injection-timing. For deviations $\Delta \beta$ and $\Delta \omega$ of the particle velocity and the frequency from the synchronous cases the phase between particle and traveling wave will slip along the structure. The total phase slip accumulated at the end of the structure is

$$
\tau=\frac{L \Delta \omega}{v_{g}}\left(1-\frac{v_{g}}{v_{p}}\right)
$$

and consists of a contribution from the dispersive characteristic of the TWS and from the nonsynchronous particle velocity. By integration over the electric field along the structure including the phase slip $\tau$, the effective accelerating voltage seen by the beam is

$$
V_{r f}=E_{0} e^{j \Phi} L \underbrace{\frac{\sin (\tau / 2)}{\tau / 2}}_{\text {TTF }}
$$

including the so-called TTF for the particle transit [6]. Let $R$ denote the shunt impedance per unit length of the TWS. Commonly, also the so-called series impedance $R_{2}=R / l_{0}$ is defined that relates the accelerating field and the power flux in the structure. The accelerating voltage is then expressed via the generator current $I_{g}$ and the forward transfer impedance $Z_{r f}$ as

$$
V_{r f}=\underbrace{L \sqrt{\frac{R_{2} Z_{0}}{2}} \frac{\sin (\tau / 2)}{\tau / 2}}_{Z_{r f}} I_{g} e^{j \Phi} .
$$

$Z_{0}$ designates the characteristic impedance of the rf system, typically $50 \Omega$. The TTF limits the bandwidth of the structure usable for particle acceleration.

The waves excited in each cell by the beam will travel in synchronism with the beam along the structure at the frequency at which particle and phase velocity are identical. The waves add linearly in the beam-forward direction, whereas on-average they cancel out in the reverse direction. The decelerating electric field seen by the beam is then simply proportional to the length of the structure measured from the rf input end. In general, the beam induced electric field $E_{b}(z)$ along the axis $z$ of the structure is

$$
E_{b}(z) \propto \frac{1-e^{-j \frac{\tau}{L} z}}{j \frac{\tau}{L}} .
$$

Note that the beam induced field is zero at the end of the TWS connected to the generator at $z=0$, which does not see the beam and therefore always remains matched. By integrating $E_{b}(z)$ along the structure the total, self-induced voltage $V_{b}$ on the beam is obtained to

$$
V_{b} \propto \frac{L^{2}}{2}\left[\left(\frac{\sin (\tau / 2)}{\tau / 2}\right)^{2}-2 j \frac{\tau-\sin (\tau)}{\tau^{2}}\right] .
$$

With the series impedance $R_{2}$ the beam coupling impedance of a TWS is given by [14]

$$
Z_{b}=\frac{R_{2} L^{2}}{8}\left[\left(\frac{\sin (\tau / 2)}{\tau / 2}\right)^{2}-2 j \frac{\tau-\sin (\tau)}{\tau^{2}}\right] .
$$

Note that the beam coupling impedance scales with the length of the structure squared. The total voltage $V$ seen by a beam passing through a TWS is the superposition of $\mathrm{rf}$ voltage and self-induced beam loading following

$$
V=Z_{r f} I_{g}-Z_{b} I_{b}
$$

\section{MEASUREMENT OF TRANSIT TIME FACTOR}

\section{A. Theory}

The frequency dependence of the rf voltage is entirely contained in the TTF, see Eq. (3). The $\sin (x) / x$-behavior could be reconstructed by sampling the frequency range with nonresonant perturbation measurements. It is shown in the following, that alternatively the TTF can be obtained from measurement samples in each accelerating gap. Opposed to perturbation techniques, the voltage can however certainly not be measured in absolute terms as in practice measurement probes must be situated off-axis.

The accelerating $\pi / 2$ mode consists of the superposition of an infinite number of traveling-wave space harmonics [15]. However, only a single space harmonic, the one with a phase velocity equal to the particle velocity, is effective in particle acceleration. We can therefore start our analysis by describing merely this synchronous (backward) travelingwave space harmonic injected by the generator with

$$
E^{+}(z, t)=E_{0} e^{-j k z} e^{j \omega t},
$$

for once also explicitly writing the time harmonic dependence. We can measure this traveling wave at $N$ off-axis 


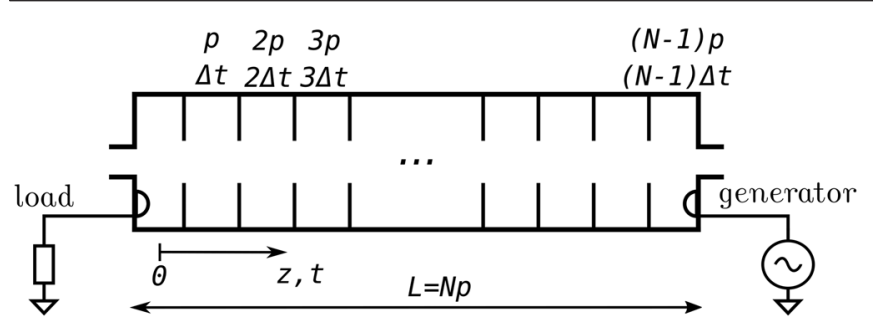

FIG. 3. Schematic view of a TWS providing access ports for $\mathrm{rf}$ measurements in each accelerating gap at the positions $z=n p$, $n=0,1, \ldots N-1$. The beam passes the center of each gap at the time $t=n \Delta t$.

sampling points in the gap centers at $z=n p$, see schematic in Fig. 3, obtaining field strength readings proportional to the values on-axis in the respective gaps. In passing through all gaps, the beam experiences merely time-shifted versions of our measurement samples and so we write the measured voltage $V_{r f, m}$ as

$$
V_{r f, m} \propto \sum_{n=0}^{N-1} E_{0} p e^{-j k n p} e^{j \omega n \Delta t} .
$$

Here we assumed a constant electric field across each gap and $\Delta t=p / v_{p}$ is the time it takes the beam to pass from one gap to the next. We use a linear expansion around the synchronous point $\left(\omega_{0}, k_{0}\right)$ of the dispersion curve

$$
\begin{aligned}
\omega & =\omega_{0}+\Delta \omega \\
k & =k_{0}+\frac{d k}{d \omega} \Delta \omega=k_{0}+\frac{\Delta \omega}{v_{g}}
\end{aligned}
$$

to express the exponent in Eq. (9) via the phase slip $\tau$ defined in Eq. (2). The time shift for the backward $\pi / 2$ mode at synchronism is $\Delta t=\pi / 2 \omega_{0}$. We reformulate the exponent in Eq. (9) to

$$
\begin{aligned}
k n p-\omega n \frac{\pi / 2}{\omega_{0}} & =n\left(\frac{\Delta \omega}{v_{g}} p-\frac{\pi}{2} \frac{\Delta \omega}{\omega_{0}}\right) \\
& =\frac{n p}{v_{g}} \Delta \omega\left(1-\frac{\pi / 2}{p} \frac{v_{g}}{\omega_{0}}\right) \\
& =: \frac{n \tau}{N} .
\end{aligned}
$$

The geometric series becomes

$$
V_{r f, m} \propto E_{0} p \sum_{n=0}^{N-1} e^{-j \frac{n \tau}{N}}=E_{0} p \frac{1-e^{-j \tau}}{1-e^{-j \frac{\tau}{N}}}
$$

We now separate modulus and phase and obtain for what we measure

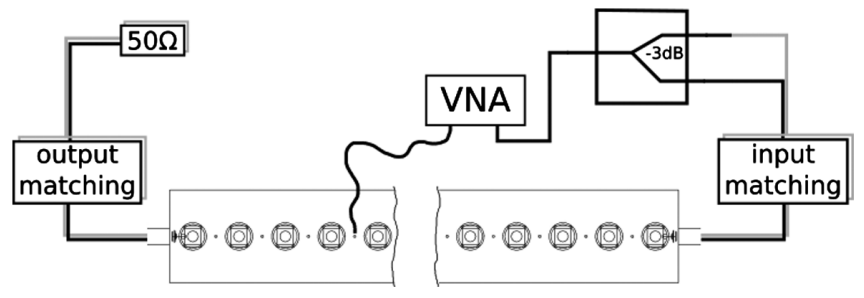

FIG. 4. Setup for the measurement of rf voltage in a TWS. One port of the VNA is used to inject a traveling wave and the second port to probe the wave in each gap. The coaxial lines in front of the input matching network are equipped with adapters to the type $\mathrm{N}$ connector standard. A $3 \mathrm{~dB}$ combiner is used to simultaneously drive the two input ports of the structure. Note that power coupling is carried out via the loops left and right to the beam pipes.

$$
V_{r f, m} \propto E_{0} L e^{j \phi} \frac{\sin (\tau / 2)}{N \sin (\tau / 2 N)} .
$$

When comparing to Eq. (3), the voltage actually seen by the beam, we notice that the TTF differs only in the denominator. The difference will become significant for $\tau / 2 N>\pi / 4$, so for $\tau>N \pi / 2$. This is merely the case for very large $\Delta \omega$, e.g., $\tau(\Delta \omega=1 \mathrm{MHz})=0.53 \mathrm{rad}$ only. The error manifests in amplitude. We can therefore measure the TTF of the forward impedance by summing time shifted measurement samples taken in the accelerating gaps of a TWS. It should be mentioned that an rf feedback system implementing the summation of cell signals similar to Eq. (9) was first proposed in [16] for compensation of beam loading.

\section{B. Application to the SPS traveling wave structures}

With the previously described technique it is possible to measure the rf voltage in a TWS around its synchronous frequency. The method is applied in the following to the four TWSs used in the SPS until the end of 2018, i.e., before the start of the upgrade works. The setup for the measurement of the TTF is illustrated in Fig. 4. A vector network analyzer (VNA) is used to inject a traveling wave. The coaxial feeder lines connecting the rf generators to the input matching network are replaced by adapters to the type $\mathrm{N}$ connector standard. The two input ports of the structure are driven simultaneously via a $3 \mathrm{~dB}$ combiner. The traveling wave is probed with the second port of the VNA in each gap of the structure via the lateral access ports shown in Fig. 2. Modulus and phase of the transmission $S_{21}$ across the frequency range of the FPB are recorded in each cell for data postprocessing later on.

Most precise results would be obtained by using the same measurement probe in each lateral port or a set of previously calibrated probes. It is well known that even probes of the same type differ slightly in their rf characteristics, which could lead to a falsification of the phase advance per cell of the traveling wave measured in 


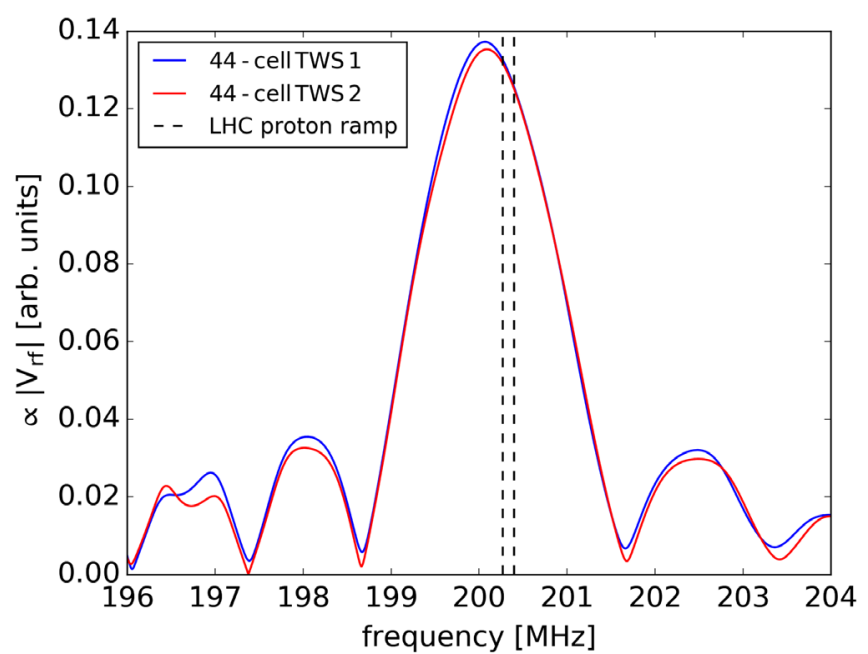

(a) 44-cell TWSs.

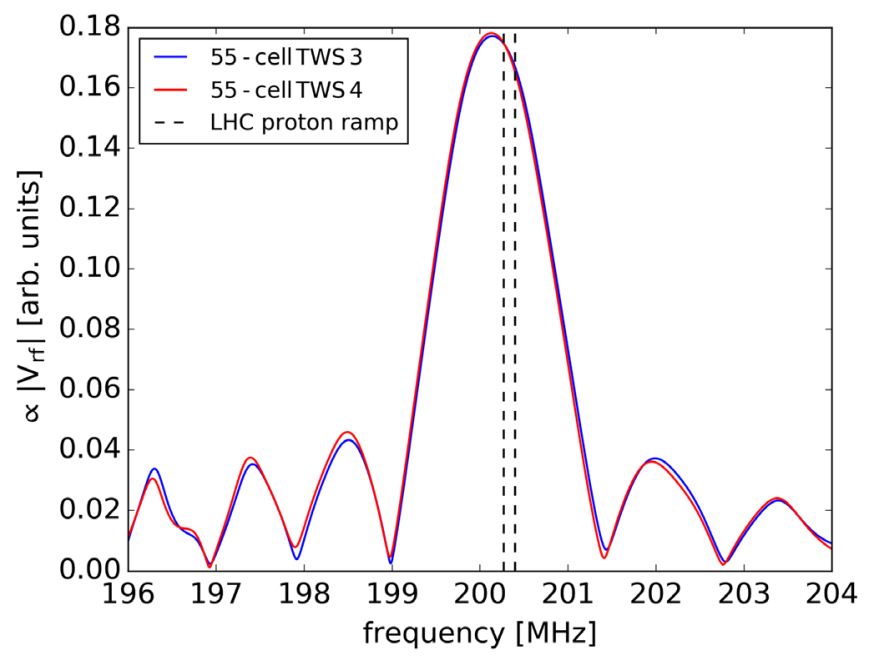

(b) 55-cell TWSs.

FIG. 5. Measurement of frequency dependence of rf voltage in the four SPS TWSs in operation until before the upgrade with the longitudinal and transverse HOM-couplers in place. The synchronous frequencies of the TWSs 1 to 4 are $200.063 \mathrm{MHz}$, 200.089 MHz, 200.136 MHz, and 200.133 MHz, respectively. The dashed, vertical lines highlight the frequency range required for ramping the LHC proton beam in the SPS $(200.265 \mathrm{MHz}$ to 200.394 MHz).

consecutive cells. For economical reasons the lateral loop couplers already in place on the SPS TWSs are used. Prior to this, the transmission to a small number of lateral loops was measured in the same cell. This showed a phase difference within less than a degree so that the accuracy of our measurements with noncalibrated pickups should be satisfactory.

The measurement data is time-shifted and summed according to Eq. (9). The results for the four SPS TWSs, of which two consist of four and two of five 11cell sections, are shown in Fig. 5. The $|\sin x / x|$ characteristic of the TTF is easily recognized as well as the smaller
TABLE I. Available rf voltage at the extraction frequency of the LHC proton ramp in the SPS TWSs 1 to 4 in percent of maximum due to detuning as well as in- and estimated output mismatch.

\begin{tabular}{lcccc}
\hline \hline & \multicolumn{3}{c}{ Contribution of... } \\
\cline { 2 - 4 } TWS & & Input & Output & Available \\
\hline$\#$ & Detuning & Mismatch & Mismatch & $V_{r f}$ \\
\hline & {$[\%]$} & {$[\%]$} & {$[\%]$} & {$[\%]$} \\
\hline 1 & 91.5 & 99.0 & 86.2 & 78.1 \\
2 & 92.5 & 99.2 & 88.3 & 81.0 \\
3 & 92.9 & 99.8 & 93.0 & 86.2 \\
4 & 92.9 & 100 & 99.2 & 92.2 \\
\hline \hline
\end{tabular}

bandwidth of the longer 55-cell structures. The TWSs were originally tuned to $200.222 \mathrm{MHz}$, however we observe the peak impedance of all four structures at significantly lower frequencies. This means that the rf voltage during the ramp of the LHC proton beam in the SPS from $200.265 \mathrm{MHz}$ to $200.394 \mathrm{MHz}$ is even lower than what was to be expected. The loss in rf voltage at extraction due to this detuning is listed in Table I at the end of this paper. A possible explanation for this detuning are the numerous HOMcouplers of the longitudinal and transverse damping schemes added after the original tuning to $200.222 \mathrm{MHz}$. This is supported by the fact that we see a significant difference in synchronous frequencies of TWSs 1 and 2 of $26 \mathrm{kHz}$ while TWS 1 is equipped with two transverse HOMcouplers more than TWS 2 . TWSs 3 and 4 are equipped with identical numbers and types of HOM-couplers and show peak impedance at quasi-identical frequencies, see Fig. 5(b). The possible impact of the HOM-couplers on the frequency dependence of the accelerating voltage is investigated in further detail in the following Sec. IV. The amplitude difference visible in Fig. 5(a) is most likely due to lateral loops with slightly different rf characteristics.

\section{IMPACT OF HOM-COUPLERS ON THE RF VOLTAGE}

Additional HOM-couplers have to be placed on the 3 -section structures to improve mode damping around $630 \mathrm{MHz}$. On the 4-section structures the desired mitigation can nearly be achieved by improvement of the existing couplers [8]. To study the impact on the fundamental accelerating mode, eight HOM-couplers were added on the 4-section TWS 2 as a 3-section configuration was not available for this measurement. Figure 6 compares the TTF of TWS 2 before and after the addition of the HOMcouplers and shows a detuning to lower frequencies by $85 \mathrm{kHz}$. This can be explained by the fact that all HOMcouplers are placed in regions with strong electric field of the fundamental mode. Figure 7 shows the additional loss in rf voltage provided by the 4-section TWS 2 due to a further detuning by $-85 \mathrm{kHz}$ in the frequency range 


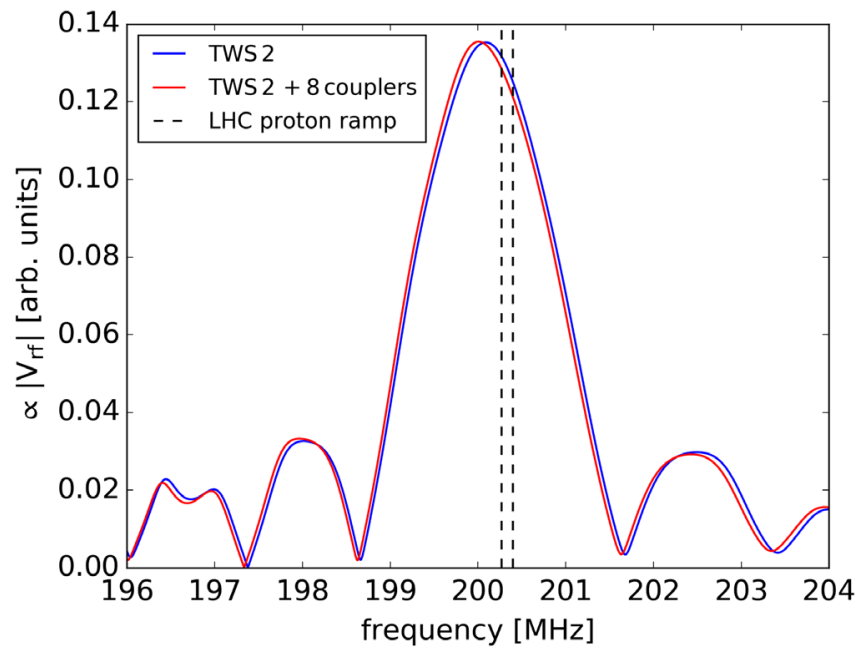

FIG. 6. Measurement of frequency dependence of rf voltage in the SPS 44-cell TWS 2 with and without eight additional HOM-couplers. The synchronous frequency is shifted by $-85 \mathrm{kHz}$ to $200.004 \mathrm{MHz}$ due to the additional HOM-couplers. The dashed, vertical lines highlight the frequency range required for ramping the LHC proton beam in the SPS $(200.265 \mathrm{MHz}$ to $200.394 \mathrm{MHz}$ ).

required for the ramp of the LHC proton beam. The loss in rf voltage amounts to roughly $2.5 \%$ at injection and $3.5 \%$ at the flat top of $450 \mathrm{GeV}$. The improved stability of the LHC proton beam obtained from the increased HOM damping outweighs however the effects of the reduced accelerating voltage [17]. Figure 7 does not however show the amount of rf voltage which is already lost due to the existing HOM damping scheme nor due to the fact that the structures are intrinsically tuned below the frequency range of the ramp. For a 3-section structure originally as well tuned to

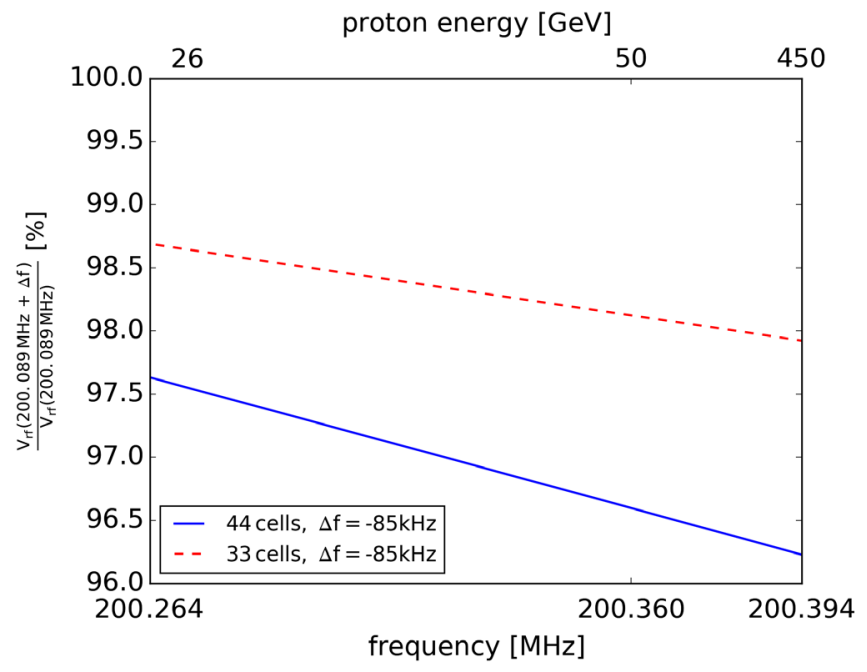

FIG. 7. RF voltage $V_{r f}$ provided by 44- and 33-cell TWSs detuned by $-85 \mathrm{kHz}$ with respect to structures tuned to 200.089 MHz in the frequency range required for the ramp of the LHC proton beam from $26 \mathrm{GeV}$ to $450 \mathrm{GeV}$ in the SPS.
200.089 $\mathrm{MHz}$ and then undergoing a detuning by $-85 \mathrm{kHz}$, the loss in rf voltage is less severe due to its larger bandwidth, cf. Fig. 7. Damping of the fundamental mode due to the HOM-couplers can be neglected as these are either fitted with well tuned notch filters or do not couple to the fundamental mode [8]. Adding HOMcouplers also influences the in- and output match. Matching of the six TWSs in the new configuration will therefore be done with all HOM-couplers in place.

\section{IMPACT OF GENERATOR AND LOAD MISMATCH ON RF VOLTAGE}

Proper traveling wave operation and a waveguide-like behavior of a TWS is ensured by matching networks at the in- and output. The input network matches the structure to the rf generator and avoids excessive reflection of power. A mismatch results in less power transfer to the TWS and consequently a lower accelerating voltage. The quality of the match can simply be measured by the input reflection coefficient $S_{11}$. With the series impedance $R_{2}$ and the input power $P$, we rewrite Eq. (3) for the voltage induced by the rf generator to

$$
V_{r f}=\sqrt{R_{2} P} e^{j \Phi} L \frac{\sin (\tau / 2)}{\tau / 2} .
$$

The actual rf voltage taking the input-mismatch into account is then reduced to

$$
\sqrt{1-\left(S_{11}\right)^{2}} V_{r f}
$$

The output network matches the terminating load to the TWS, thus avoiding reflections at the end of the structure back toward the rf input. The consequence of a load mismatch is briefly studied in the following also with the goal of finding an appropriate criterion to assess the quality of the match. Consider the schematic of a TWS with spatial period $p$ and load mismatch $\Gamma$ at $z=L$ in Fig. 8. The generator injects the traveling wave

$$
E^{+}=E_{0} e^{-j k z}
$$

with wave number $k=\Delta \varphi / p$ into the $N$-cell structure. The wave reflected at the $\mathrm{rf}$ output at $z=L=N p$ is

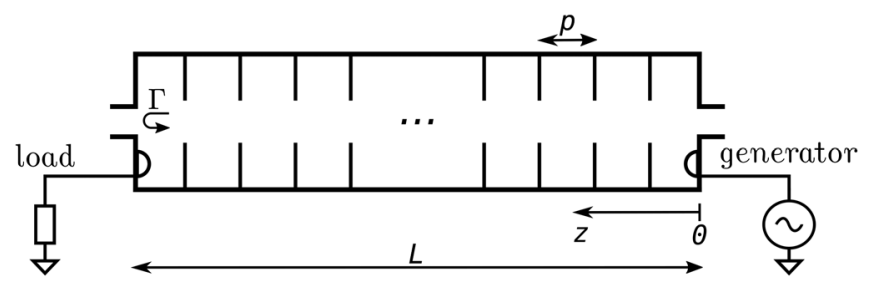

FIG. 8. Schematic view of a traveling wave system with load mismatch $\Gamma$ and spatial period $p$. 


$$
E^{-}=\Gamma E_{0} e^{j k(z-2 L)}
$$

We neglect attenuation and a rereflection at the rf input which usually is a very good approximation. For the case of a $\pi / 2$-mode, we write the superposition $\Sigma=E^{+}+E^{-}$as

$$
\begin{aligned}
\Sigma_{\pi / 2}= & E_{0}(1-\Gamma) e^{-j \frac{\pi}{2 p} z} \\
& +E_{0} 2 \Gamma \begin{cases}\cos \left(\frac{\pi}{2 p} z\right) & \text { for } \mathrm{N} \text { even } \\
-j \sin \left(\frac{\pi}{2 p} z\right) & \text { for } \mathrm{N} \text { odd. }\end{cases}
\end{aligned}
$$

This is a forward traveling wave of reduced amplitude $E_{0}(1-\Gamma)$ with a superimposed $\pi / 2$ standing wave of amplitude $E_{0} 2 \Gamma$. The $\pi / 2$ standing wave also exists in a TWS with uneven number of cells $N$ as we assumed no rereflected wave. The phase of $\Gamma$ will depend on the output matching network. If $\Gamma$ is of zero phase at $z=L$, Eq. (13) shows that the standing wave has no net accelerating effect on the beam. Ignoring the anyway unwanted standing wave, the actual rf voltage $\tilde{V}_{r f}$ obtained taking the generator and load mismatch into account is

$$
\tilde{V}_{r f}=\sqrt{1-\left(S_{11}\right)^{2}}(1-\Gamma) V_{r f}
$$

A good match of a TWS to its load is more important than its input match. The $\pi / 2$ standing wave manifests as a $\pi$ pattern with amplitude $E_{0} \Gamma$ on the modulus of the total field along the TWS, refer to Sec. VII C.

\section{MATCHING OF TRAVELING WAVE STRUCTURES}

In case of waveguide-fed TWSs, matching is usually obtained by special coupling cells at the in- and output and numerous design procedures have been proposed, see, e.g., [18]. The $200 \mathrm{MHz}$ structures in the SPS however are connected via $50 \Omega$ coaxial lines and the inner conductors end in coupling loops on the pedestals (see Fig. 1) of the first and last half cells. In this case, in-line matching networks are deployed to match the rf generator and the terminating load to the structure. Another important task of the matching is damping of high-impedance modes adjacent to the accelerating $\pi / 2$ mode. An inherent broadband match of the coupling loops is therefore paramount as otherwise the beam would drive itself unstable due to standing wave resonances in the FPB.

For matching of the SPS TWS in EM simulations and for rf measurements on a 33-cell test structure, the simultaneous complex conjugate matching (SCM) procedure was applied [8]. To the authors knowledge this approach has so far not been used for matching TWSs, but it is well known from, e.g., transistor amplifier design $[19,20]$. Maximum power shall be transferred from the generator to the TWS.
A pure traveling wave in the structure is ensured if maximum power is also transferred to the load at the rf output. For a one-port network it is well known that maximum power transfer is obtained through complex conjugate matching at the port. The output of the unmatched stem-loaded waveguide acts back on its input, which is why the addition of an output matching network would change the input reflection coefficient of the structure and vice versa. With the SCM, in- and output networks can be designed simultaneously to ensure complex conjugate match at both ports. Another advantage is that only a single measurement of the scattering parameters is required to design both networks in one step. This is in direct contrast to iterative methods that adjust the matching networks until no reflection is observed measuring into the in- and output networks. In addition, it then has to be verified by other means that the apparent match is not merely the result of a fortuitous cancellation of forward and backward waves in the accelerating structure at the position of the couplers. The SCM procedure is applied in Sec. VII C to the EM simulation of a 33-cell structure.

\section{THE SPS 200 MHz PARALLEL STEM DRIFT-TUBE STRUCTURE}

A traveling wave system has two major advantages. The power amplifiers, which always see a matched load, can be located far away in a surface building where they can easily be maintained and expanded, as it is the case with the present upgrade. In addition, a mechanical tuner is not required, making the structures highly reliable due to the absence of any moving parts. The TWSs are fixed-tuned and operate over a wide bandwidth. At first sight a disadvantage of TWSs is the large power consumption compared to standing wave cavities. However, this fact becomes negligible for high beam intensities with strong beam loading, which changes the spatial distribution of electric field along the structure, see Eq. (5). A TWS then has the particularity that most of the additionally required power for restoring the accelerating field to its desired value does not equal the beam power, but results mostly from a decrease of the power that is otherwise lost in the terminating load.

\section{A. Dispersion characteristics}

The dispersion characteristics of an infinite periodic structure can conveniently be obtained from the simulation of its unit-cell with periodic boundaries [21]. Figure 9 shows the first three passbands in the SPS TWS obtained from such unit cell simulations. In addition to the usual passbands of the unloaded, uniform waveguide, a backward-wave passband is created below the cutoff frequency of the unloaded guide. It is called the stem-passband as it results from the stems that behave as coupled resonators. The "lightline" in Fig. 9 suggests that the beam is 


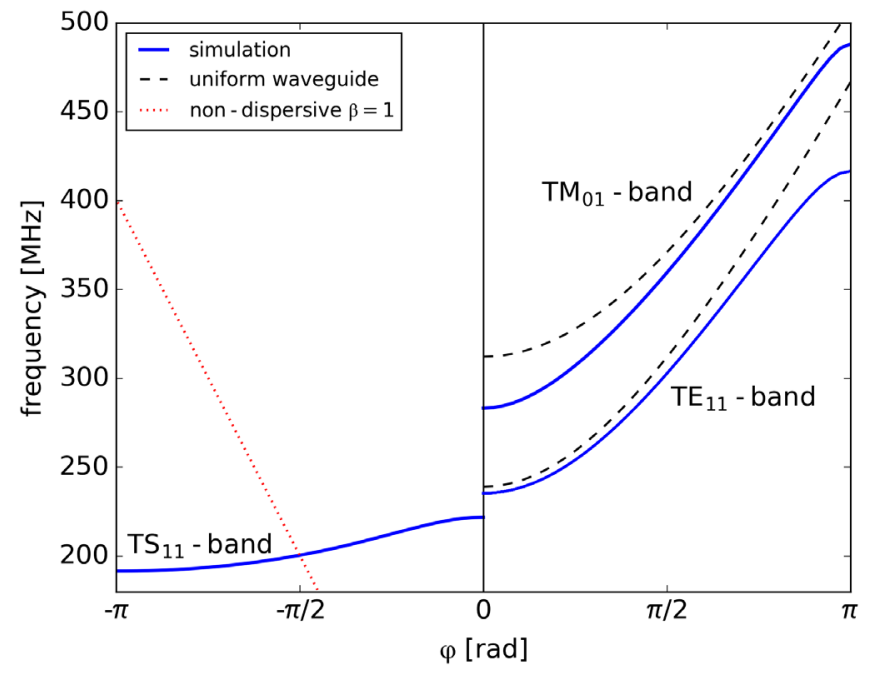

FIG. 9. Fundamental space harmonics of the three lowest passbands in the SPS TWS. The beam (dotted) is synchronous with the fundamental stem mode $\mathrm{TS}_{11}$ at $200.222 \mathrm{MHz}$ and a phase advance per cell of $\varphi=-\pi / 2$. Refer to [8] for field profiles and mode notation. The analytic calculation of the $\mathrm{TE}_{11}$ and $\mathrm{TM}_{01}$ bands in the corresponding unloaded circular waveguide is given as reference (dashed) [22].

synchronous with the stem mode at around $200.222 \mathrm{MHz}$ having a $-\pi / 2$ phase advance per cell. The $\pi / 2$-mode was chosen for operation as it features high shunt impedance and approximately the desired group velocity.

The $R / Q$ of the structure at the traveling-wave operating mode is simulated to $626 \Omega / \mathrm{m}$, the attenuation length to $515 \mathrm{~m}$ and consequently the series impedance is $27.8 \mathrm{k} \Omega / \mathrm{m}^{2}$. These values are only slightly higher than those obtained by measurements and given in [6].

\section{B. Impedance calculation of 33-, 44-, and 55-cells}

The phase and group velocities in the frequency range of the FPB are easily calculated from its simulated dispersion curve and plotted in Fig. 10. Note the opposite signs due to the backward characteristic of the stem passband. The respective values at $200.222 \mathrm{MHz}$ are $v_{p} \approx c$ and $v_{g}=0.09466 c$. Using these frequency dependent phase and group velocities we can calculate the forward and beam coupling impedances of a TWS with an arbitrary number of cells in the frequency range of the FPB. The forward impedance $Z_{r f}$ of 33-, 44-, and 55-cells is plotted in Fig. 11 according to Eq. (4). Peak impedance is provided by the structures at the synchronous frequency for which the phase slip between EM wave and particle beam is zero along the whole length of the structure. The usable bandwidth is limited by the sinc-characteristic of the TTF. Note the asymmetric distribution of the zeros about the synchronous frequency and the more broadband characteristic of shorter structures. The $-3 \mathrm{~dB}$ bandwidths are 1.2, 1.4, and $1.9 \mathrm{MHz}$ respectively.

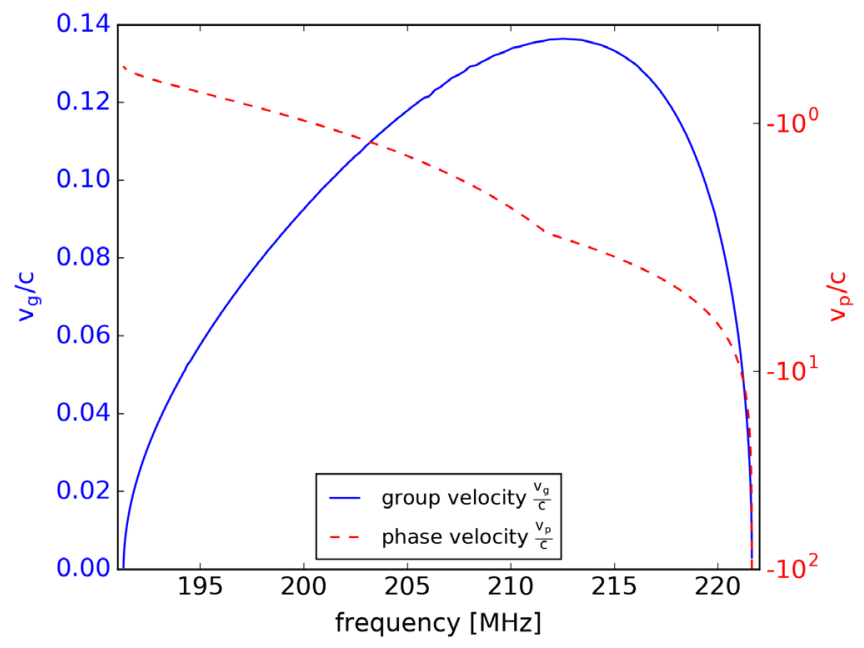

FIG. 10. Simulated group velocity $v_{g}$ and phase velocity $v_{p}$ of the SPS TWS in the frequency range of the FPB.

The beam coupling impedance $Z_{b}$ of 33-, 44-, and 55cells is plotted in Fig. 12, following Eq. (7). Note the considerably lower peak coupling impedance of 33-cell structures compared to that of 55-cells.

\section{Simulation of $\mathrm{rf}$ voltage in 33-cells}

In simulations, we have direct access to the on-axis electric field and can easily confirm our findings of Sec. V. For illustration we compare a well matched 33-cell structure realized using the SCM technique from Sec. VI with a significantly mismatched structure. In the latter case the feeder lines were terminated at an arbitrary distance to the coupling loops by their $50 \Omega$ characteristic impedance instead of the impedance required for proper matching. The reflection and transmission coefficients in the two

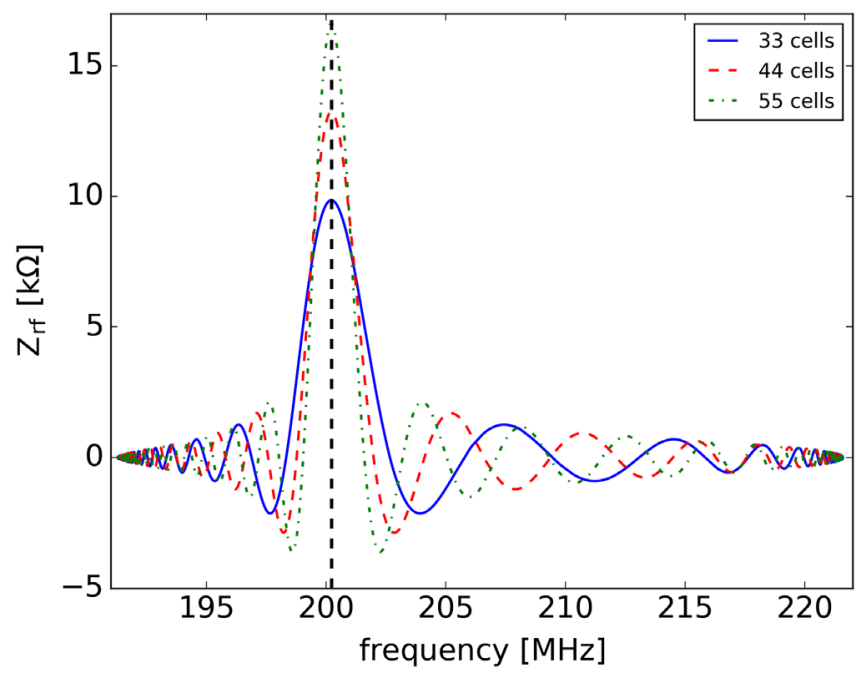

FIG. 11. Simulated forward transfer impedance $Z_{r f}$ of 33-, 44-, and 55-cell SPS TWSs tuned to $200.222 \mathrm{MHz}$ in the frequency range of the FPB. 


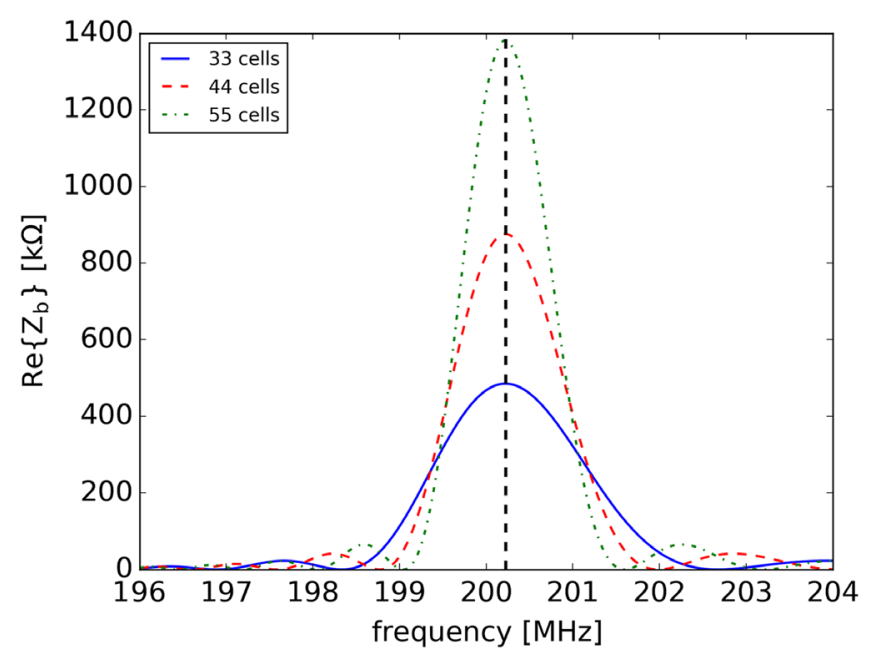

(a) Real part.

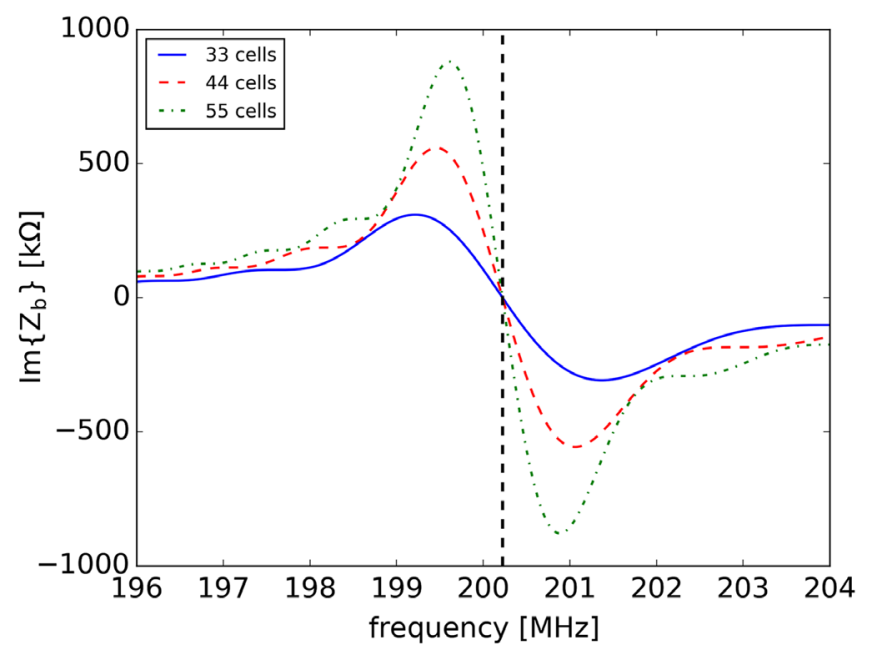

(b) Imaginary part.

FIG. 12. Simulated beam coupling impedance $Z_{b}$ of 33-, 44-, and 55-cell SPS TWSs tuned to $200.222 \mathrm{MHz}$ in the frequency range of the FPB.

cases are compared in Fig. 13. The reflections at the synchronous frequency of $200.222 \mathrm{MHz}$ amount to $S_{11}=$ $-4.1 \mathrm{~dB}$ and $S_{11}=-30.1 \mathrm{~dB}$ respectively. In simulation, $S_{11}=S_{22}$ and $S_{21}=S_{12}$ due to the symmetry of the model. From the scattering parameters of the mismatched case, the SCM technique provides immediately and without requiring any further adjustments the impedance of the matching networks that leads to the excellent match shown in Fig. 13(a). A narrowband match around 200.222 MHz resulting in negligible reflections toward the rf source is obtained as well as the broadband characteristic required to damp adjacent, high-impedance modes excited by the beam. Figure 13(b) illustrates the bandpass like behavior of the structure. In the well matched case and for an $\mathrm{rf}$ injected around $200 \mathrm{MHz}$, the 33-cell structure basically behaves as a waveguide with the transmission $S_{21}=$ $-0.12 \mathrm{~dB}$ being quasi entirely a result of its resistive

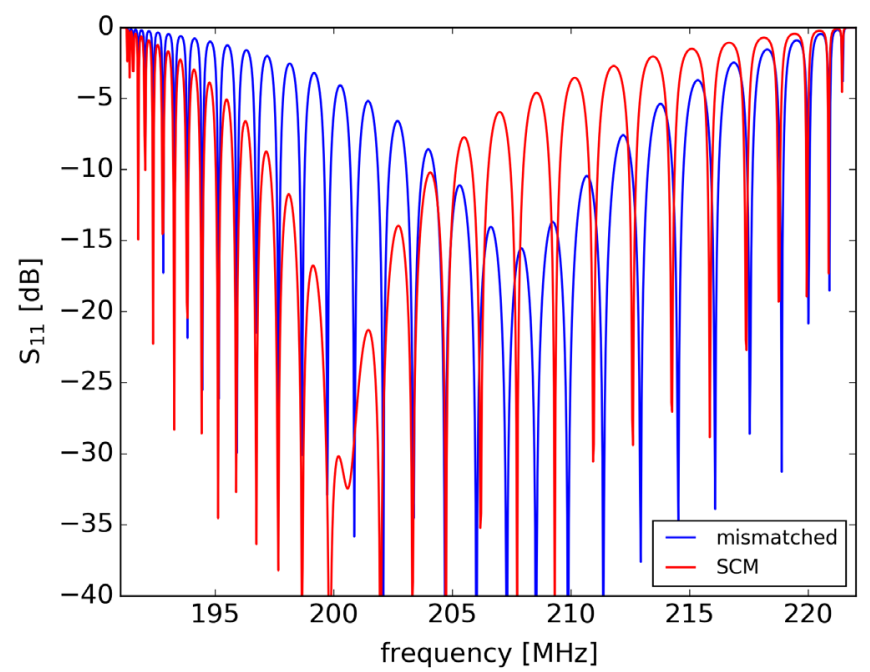

(a) Reflection $S_{11}$

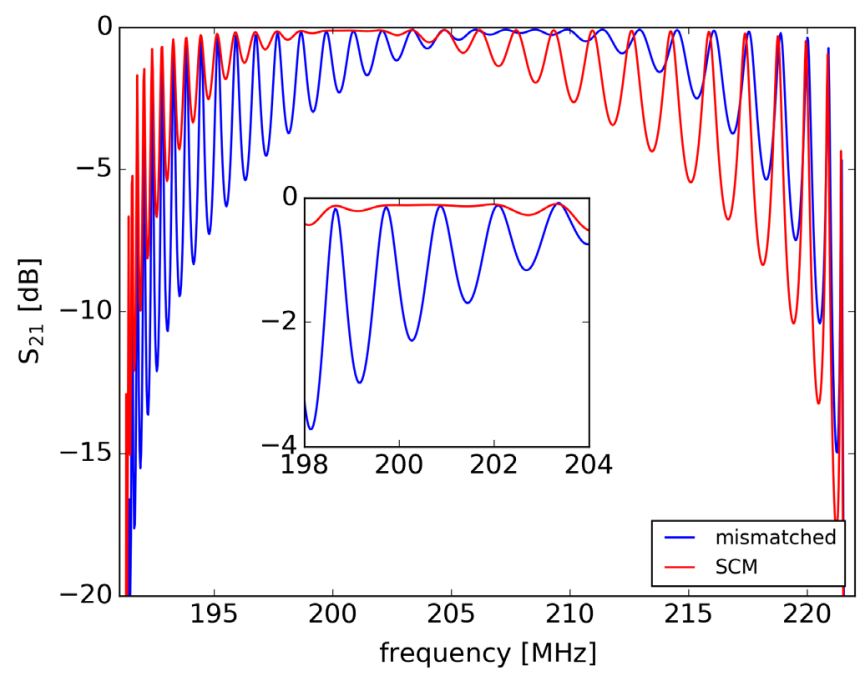

(b) Transmission $S_{21}$.

FIG. 13. Comparison of scattering parameters for the simulated 33-cell structure with arbitrary match and simultaneous complex conjugate match (SCM). The SCM results in a broad- and narrowband match around the synchronous frequency of 200.222 MHz. Due to the exact symmetry in simulations $S_{11}=$ $S_{22}$ and $S_{21}=S_{12}$.

losses. The typical characteristics of resonant modes are no longer visible in this frequency range as they are damped by the coupling loops with the attached SCM networks.

The electric field on the central axis of the 33-cell structure at $200.222 \mathrm{MHz}$ is shown in Fig. 14 for the two matchings. The modulus of the electric field clearly shows the $\pi$-pattern due to the mismatch of the terminating load to the TWS as predicted by the analysis in Sec. V. Using the Kroll method [23], we can determine the output coupler mismatch to $\left|\Gamma_{\text {arb }}\right| \approx S_{22}=-4.1 \mathrm{~dB}$ from the electric field in the structure. It is convenient that in case of the SPS TWS the load match can also simply be determined from a $S_{22}$ reflection measurement. 


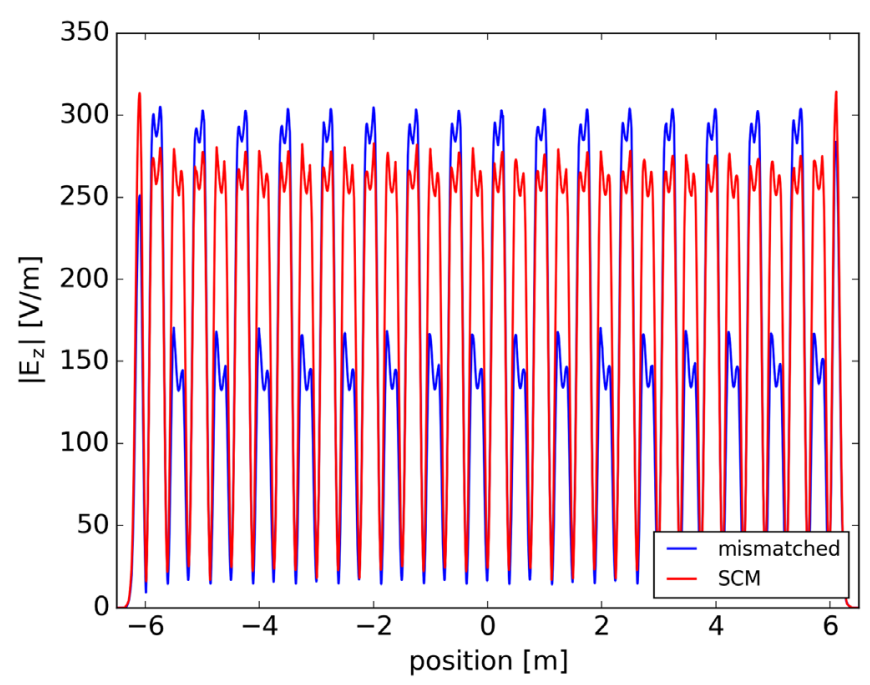

FIG. 14. On-axis, longitudinal electric field $E_{z}$ at $200.222 \mathrm{MHz}$ in the 33-cell structure with arbitrary match, $S_{11}=-4.1 \mathrm{~dB}$ ) and $\mathrm{SCM}, S_{11}=-30.1 \mathrm{~dB}$ ) for an input power of $1 \mathrm{~W}$ peak injected at $z=+6.6 \mathrm{~m}$.

By integration of the on-axis electric field the accelerating voltage is calculated to $597 \mathrm{~V}$ in the mismatched case and to $1.993 \mathrm{kV}$ for the SCM at $200.222 \mathrm{MHz}$. The latter is only $0.14 \%$ below the theoretical value for an input power of $1 \mathrm{~W}_{\mathrm{p}}$ and using the simulated series impedance of $27.8 \mathrm{k} \Omega / \mathrm{m}^{2}$. Instead of by integration, the accelerating voltage can also be calculated with Eq. (14) from the reflection coefficients at the in- and output. In case of the arbitrary match the voltage is obtained to $586.5 \mathrm{~V}$ by this method, which is in good agreement with the value given above.

\section{MEASUREMENT OF RF VOLTAGE IN SPS TRAVELING WAVE STRUCTURES}

To determine the absolute if voltage provided by each of the four SPS TWSs we now use the derivation in Sec. V to account for mismatch at the in- and outputs. We simply extend Eq. (14) for the $\pi / 2$-mode to the frequency range of the LHC proton ramp to obtain a good estimate of the available rf voltage from each structure. The measured output reflection $S_{22}$ is shown in Fig. 15. The input reflection $S_{11}$ on each structure is similar. For a fixed available input power, each TWS provides maximum rf voltage at its synchronous frequency, but high voltage is especially required at extraction for transfer of the proton bunches into the smaller rf buckets of the $400 \mathrm{MHz}$ LHC rf system. With Figs. 5 and 15 we determine the loss of rf voltage at extraction due to the detuning and the mismatch of generator and load with respect to the maximum voltage. Table I lists the overall estimates for the available rf voltage in each structure at extraction. The values given are in percent of the rf voltage available under ideal conditions, meaning no in- or output mismatch and the synchronous frequency tuned to extraction.

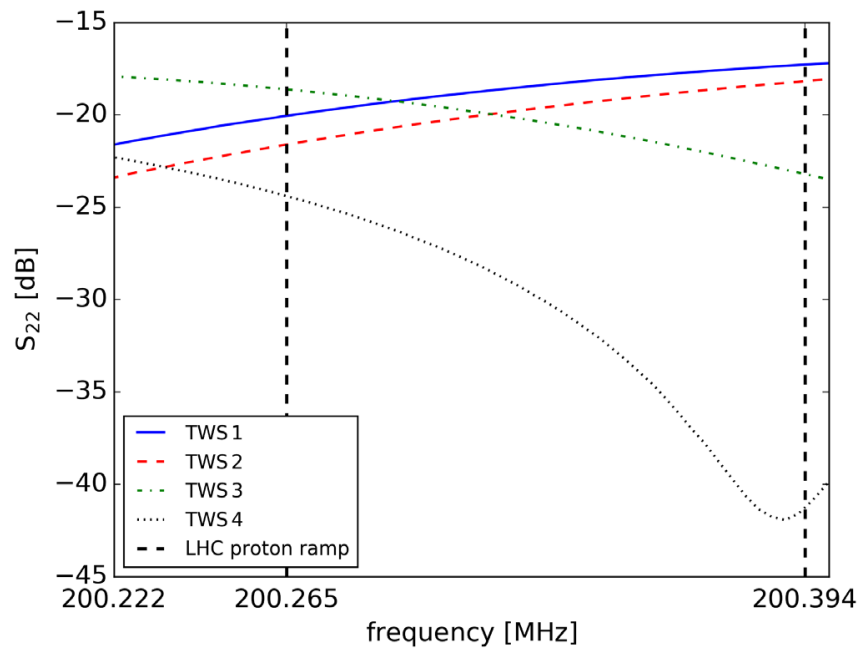

FIG. 15. Measured output reflection coefficient $S_{22}$ for the four TWSs used until LS 2 in the frequency range of the LHC proton ramp.

\section{CONCLUSIONS}

The frequency dependence of the rf voltage provided by a TWS can be measured by summing time-delayed signals obtained from each accelerating gap. The method was successfully applied to the traveling wave system while being installed in the SPS and showed that the TWSs do not provide their possible peak voltage during acceleration of the LHC proton beam. Retuning of the TWSs to higher frequencies, e.g., the extraction frequency, by shortening all stem lengths is infeasible due to the large and complicated workload. In case a retuning is required, the protrusion of metal pins through all pedestals into the area with strong magnetic field around the stems seems feasible. The additional loss in $\mathrm{rf}$ voltage due to the placement of new HOM-couplers seems acceptable at present and the shorter structures used after the upgrade are less susceptible to detuning. The measured available rf voltage is a valuable input for machine operation and particle tracking codes used to simulate beam phenomena in the SPS. It is planned to measure the rf voltage provided by the upgraded acceleration system for the High Luminosity LHC era with the proposed technique once it becomes available. If required, further studies can be conducted to include the effects of beam loading in the detuned structures. The technique to simultaneously match in- and output of a two-port network was successfully applied to the SPS TWS.

\section{ACKNOWLEDGMENTS}

The authors are grateful to A. Farricker for numerous helpful discussions and his participation in the rf measurements. Also, the authors would like to thank E. Montesinos and the whole team of BE-RF-PM for preparing the SPS 
accelerating structures for measurements. Furthermore, this work is sponsored by the Wolfgang Gentner Program of the German Federal Ministry of Education and Research (BMBF) Grant No. 05E15CHA.

[1] H. Damerau, A. Funken, R. Garoby, S. Gilardoni, B. Goddard, K. Hanke, A. Lombardi, D. Manglunki, M. Meddahi, B. Mikulec, G. Rumolo, E. Shaposhnikova, M. Vretenar, and J. Coupard, LHC Injectors Upgrade, Technical Design Report, Vol. I: Protons, CERN Technical Report CERN-ACC-2014-0337, 2014.

[2] E. Shaposhnikova, E. Ciapala, and E. Montesinos, Upgrade of the $200 \mathrm{MHz}$ RF System in the CERN SPS, Report No. CERN-ATS-2011-042, 2011.

[3] E. Shaposhnikova, T. Argyropoulos, T. Bohl, P. Cruikshank, B. Goddard, T. Kaltenbacher, A. Lasheen, J. Perez Espinos, J. Repond, B. Salvant, and C. Vollinger, Removing known SPS intensity limitations for High Luminosity LHC goals, Report No. CERN-ACC-2016289, MOPOY058, 2016.

[4] L. Smith, Linear accelerators, Encyclopedia of Physics XLIV, 341 (1959).

[5] O. H. Altenmueller, R. R. Larsen, and G. A. Loew, Investigations of traveling wave separators for the Stanford 2-mile linear accelerator, Rev. Sci. Instrum. 35, 438 (1964).

[6] G. Dôme, The SPS acceleration system travelling wave drift-tube structure for the CERN SPS, Report No. CERNSPS-ARF-77-11, 1977.

[7] G. Dôme and I. White, A general theory of multistem drift tube structures proposed for proton linacs, Report Nos. CERN-ISR-300-LI-67-62, ISR-300-LI-67-62, 1967.

[8] P. Kramer, Studies of higher order mode couplers for the upgraded travelling wave acceleration system in the CERN SPS, Ph.D. thesis, CERN, Institute of High Frequency Technology (IHF), RWTH Aachen University, Germany (to be published).

[9] J. Repond, Possible mitigations of longitudinal intensity limitations for HL-LHC beam in the CERN SPS,
Ph.D. thesis, CERN, Ecole Polytechnique Federale de Lausanne, Switzerland (to be published).

[10] P. Kramer and C. Vollinger, Improved HOM-mitigation for future SPS 33-Cell $200 \mathrm{MHz}$ traveling-wave accelerating structures, Int. J. Mod. Phys. A (to be published).

[11] N. Nasresfahani, HOM damping in accelerating cavities with large number of cells: Application to 44-Cell TWCs of CERN SPS, https://indico.classe.cornell.edu/event/185 (2018).

[12] C. Steele, A nonresonant perturbation theory, Report No. SLAC-TN-64-001, 1964.

[13] The design study of a $300 \mathrm{GeV}$ proton synchrotron (CERN, Geneva, 1964), pp. 113-135.

[14] D. Boussard, Beam loading, Report No. CERN-1987-003V-2.626 (1985).

[15] J.C. Slater, Microwave Electronics (D. van Nostrand Company, New York City, 1950).

[16] D. Boussard, Possible ways of improving the longitudinal behaviour of the high intensity beam at injection in the SPS, CERN Technical Report Nos. SPSARF-Technical-Report-DB-gs-81-2, CERN-SPS-ARF81-2, 1981.

[17] J. Repond and E. Shaposhnikova, Effect on beam stability of $200 \mathrm{MHz}$ voltage reduction, https://indico.cern.ch/ event/788200/ (2019).

[18] P. M. Lapostolle and A. L. Septier, Linear Accelerators (North-Holland, Amsterdam, 1970).

[19] D. M. Pozar, Microwave Engineering, 4th ed. (Wiley, Hoboken, NJ, 2012).

[20] R. E. Collin, Foundations for Microwave Engineering, 2nd ed., McGraw-Hill series in electrical engineering (McGraw-Hill, New York, NY, 1992).

[21] Computer Simulation Technology (CST) AG, Darmstadt, Germany, http://www.cst.com/.

[22] C. A. Balanis, Advanced Engineering Electromagnetics (Wiley, New York, 1989).

[23] N. M. Kroll, C. K. Ng, and D. C. Vier, Applications of time domain simulation to coupler design for periodic structures, eConf C000821, TUE04 (2000). 